\title{
Parametric imaging of cancer with optical coherence tomography
}

\author{
Robert A. McLaughlin \\ Loretta Scolaro \\ University of Western Australia \\ School of Electrical, Electronic and Computer Engineering \\ Optical+Biomedical Engineering Laboratory \\ 35 Stirling Highway \\ Crawley, West Australia 6009, Australia
}

\section{Peter Robbins}

PathWes

Queen Elizabeth II Medical Centre

Nedlands, West Australia 6009, Australia

\section{Christobel Saunders}

Sir Charles Gairdner Hospital

Nedlands, West Australia 6009, Australia and

University of Western Australia

Queen Elizabeth II Medical Centre

School of Surgery

Nedlands, West Australia 6009, Australia

\section{Steven L. Jacques}

Oregon Health \& Science University

Department of Biomedical Engineering and

Department of Dermatology

3303 SW Bond Avenue

Portland Oregon 97239

\section{David D. Sampson}

University of Western Australia

School of Electrical, Electronic and Computer Engineering

Optical+Biomedical Engineering Laboratory and

Centre for Microscopy, Characterisation, and Analysis

35 Stirling Highway

Crawley, West Australia 6009, Australia

\begin{abstract}
We present a parametric optical coherence tomography (OCT) technique to improve contrast between malignant and healthy non-neoplastic tissue. The technique incorporates a fully automated method to extract tissue attenuation characteristics. Results are represented visually as a parametric en face image, where the parameter used for contrast is indicative of the relative optical attenuation coefficient of the tissue. We present the first parametric OCT images of human lymph nodes containing malignant cells, and demonstrate improved tissue contrast over en face OCT images. @ 2010 Society of PhotoOptical Instrumentation Engineers. [DOI: 10.1117/1.3479931]
\end{abstract}

Keywords: optical coherence tomography; cancer; lymph node; parametric imaging; parametric optical coherence tomography.

Paper 10310LR received Jun. 5, 2010; revised manuscript received Jul. 21, 2010; accepted for publication Jul. 22, 2010; published online Aug. 19, 2010.

\section{Introduction}

Accurate determination of the extent and spread of disease (staging) is a critical component of the care and management of individuals with cancer. The lymphatic system provides one pathway for the dissemination of malignancy in the body, and lymph nodes represent a common site of metastatic involvement in many malignancies. For staging purposes, accurate determination of the presence or absence of metastatic disease within lymph nodes requires surgical excision and microscopic examination. The removal of healthy uninvolved lymph nodes, in addition to causing additional trauma for the patient, may result in chronic complications such as lymphedema.

Address all correspondence to: Robert A. McLaughlin, University of Western Australia, School of Electrical, Electronic and Computer Engineering, Optical +Biomedical Engineering Laboratory, 35 Stirling Highway, Crawley, West Australia 6009, Australia. Tel: +61 86488 3105; Fax: +61 86488 1319; E-mail: robertm@ee.uwa.edu.au
Current clinical imaging modalities are unable to accurately predict lymph node status in many cancers, including breast cancer. However, preliminary work has demonstrated that pathological changes in lymph node morphology due to malignancy are visible under optical coherence tomography (OCT). ${ }^{1}$ Unfortunately, appearance within the OCT image varies with depth, as different tissue types attenuate the OCT signal at different rates. For this reason, healthy and malignant tissue will vary in appearance depending on location.

Other research has proposed the automatic extraction of characteristic structural features to segment tissue into classes such as adipose, stroma, and malignant tissue. ${ }^{2,3}$ In contrast to such segmentation algorithms, in this work a method to provide improved tissue contrast through the formation of a parametric OCT image is proposed and demonstrated. This improved tissue differentiation has the potential to aid both

$1083-3668 / 2010 / 15(4) / 046029 / 4 / \$ 25.00$ ○ 2010 SPIE 
manual and automated interpretation of OCT data.

Using a fully automated algorithm, attenuation characteristics are extracted from each A-scan in the OCT dataset. Results are represented visually as a parametric en face image, in which intensity is indicative of the relative optical attenuation coefficient. Earlier work has demonstrated the value of automatically extracted attenuation coefficients in OCT tissue differentiation in ophthalmology applications, ${ }^{4}$ and in the characterization of atherosclerotic plaques, ${ }^{5}$ where a color-coded representation of the attenuation coefficient was superimposed on a B-scan image. Extending on from this work, comparable en face images have been suggested for the characterization of atherosclerotic plaques in cardiac disease, although these were acquired using fixed (not fresh) tissue. ${ }^{6}$ The novelty of this work is to propose a technique for the automatic generation of such parametric en face images for the differentiation of cancerous and healthy tissue. We present the first parametric OCT images of malignant human lymph nodes, and demonstrate that such parametric images provide improved tissue contrast over normal en face OCT images. An early conference paper version of this work appeared in Ref. 7.

\section{Method}

In a turbid medium such as tissue, attenuation of an OCT signal can be modeled using a modified form of Beer's law. Reflectance $R(z)$ at depth $z$ can be expressed as follows, ${ }^{8}$

$$
R(z)=\rho \exp (-2 \mu G z)+B \exp (-C z),
$$

where $\rho$ (dimensionless) is the average reflectivity and $\mu$ $\left(\mathrm{mm}^{-1}\right)$, the observed attenuation coefficient of the tissue, is a function of both scattering and absorption. For near-infrared light in tissue, as is used in OCT, scattering is the dominant attenuation mechanism. ${ }^{9}$ The term $G$ accounts for the extra photon path length as photons are delivered obliquely toward a focus. With the low numerical apertures typically used in OCT, this factor is approximately unity. The term $B \exp (-C z)$ is a background noise floor to account for factors such as multiply scattered light. ${ }^{10}$ However, multiple scattering is not considered further in the formation of the parametric images described in this work.

We define a parametric en face OCT image in which the parameter $\mu$ is used to characterize tissue type. Given a 3-D OCT dataset, this involves a two-stage process of automatically identifying a subset of each A-scan that lies fully within the tissue, and estimating the corresponding value of $\mu$.

Identification of the appropriate subset of the A-scan utilizes image processing techniques tailored to the experimental setup. For the pilot studies undertaken here, fresh (nonfixed) tissue samples were mounted on top of a glass slide and immersed in glycerol to reduce the refractive index mismatch between the glass slide and tissue sample. Samples were then imaged through the glass slide, with the OCT probe placed beneath the sample and the light beam directed upward.

In the data recorded from such an experimental setup, each A-scan has a large peak in reflectivity early in the A-scan, corresponding to the change in refractive index as the light passes from glass into the area of tissue and glycerol. This point can be identified automatically as a local maximum in the derivative of the A-scan intensity, with the derivative approximated numerically as the difference between intensity

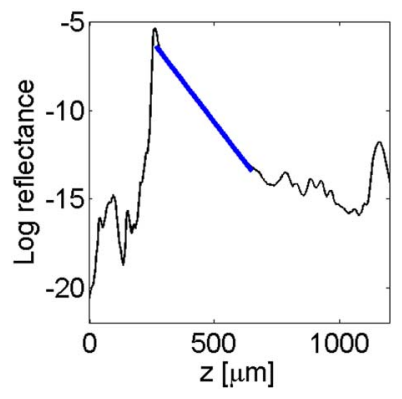

(a)

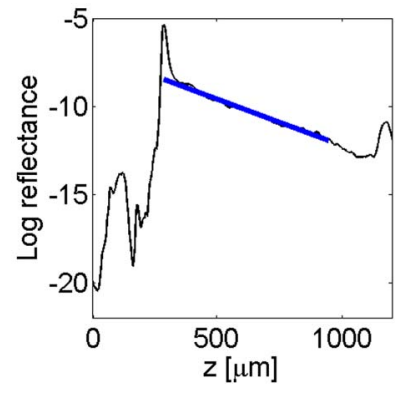

(b)
Fig. 1 Averaged A-scans from two phantom objects. The line of best fit illustrates the calculated optical attenuation. (a) phantom 1 (higher concentration of microspheres). (b) phantom 2 (lower concentration of microspheres)

values separated by a small distance. A distance of $50 \mu \mathrm{m}$ was used for all clinical datasets in these experiments. The location of this local maximum was identified for all A-scans in the acquisition, defining a set of points on the glass surface. Outliers were eliminated by robustly fitting a 2-D affine plane to this point set. The intersection of the plane with each A-scan was taken as specifying the glass interface.

Having extracted the location of the glass interface for each A-scan, the automated algorithm proceeded to identify the start of the tissue within the A-scan. At locations where the tissue did not sit flat on the glass slide, this could be several hundred microns beyond the glass interface. The start of the tissue was found by averaging A-scan values within an $80-\mu \mathrm{m}$ moving window, and identifying the location at which the smoothed OCT signal exceeded an empirically set threshold. The same threshold was used for all datasets.

To estimate the value of $\mu$ within the A-scan, the algorithm was used to extract the subset of OCT values that were within an optical path length of $0.5 \mathrm{~mm}$ of the start of the tissue. The local value of $\mu$ was then computed by taking the logarithm of these OCT values and estimating the gradient of the line of best fit using a linear least-squares fitting technique.

The value $\mu$ was independently calculated for the A-scan at each $x, y$ location, defining a 2-D map $\mu(x, y)$. This was then visualized as a parametric image, where $\mu(x, y)$ specifies the parametric image pixel value, with low values of $\mu(x, y)$ graphically represented by dark pixels and the maximum attenuation coefficient represented as a white pixel.

\section{Experiment}

To demonstrate variation in attenuation coefficients, two homogeneous microsphere phantoms were prepared to mimic scattering in tissue. Different concentrations of microspheres in suspension were used to vary the optical properties of the phantoms. The first phantom contained $0.457 \mu \mathrm{m}, 2.60 \% \mathrm{w} / \mathrm{v}$ polystyrene microspheres (Polysciences Incorporated, Warrington, Pennsylvania). The second contained one quarter of the concentration of microspheres, achieved by dilution with distilled water. The phantoms were imaged with a timedomain OCT system with central wavelength of $1320 \mathrm{~nm}$, spectral bandwidth of $150 \mathrm{~nm}$, axial resolution of $8 \mu \mathrm{m}$, and lateral resolution of $11 \mu \mathrm{m}$ in air. $512 \mathrm{~A}$-scans were acquired at the same location and averaged to reduce the effect of 

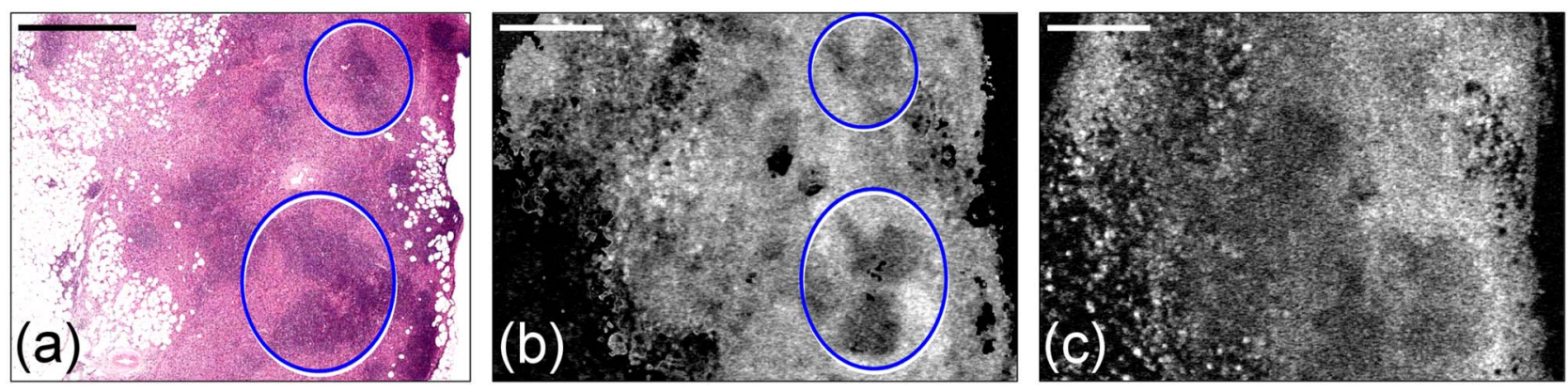

Fig. 2 Involved (malignant) human axillary lymph node with diffuse involvement of the node tissue. (a) H\&E histology; (b) parametric OCT image indicating optical attenuation coefficient of the tissue; and (c) en face OCT image. Circled areas highlight residual, noncancerous cortical tissue. Scale bar $=1 \mathrm{~mm}$.

speckle noise. The attenuation coefficient was estimated by finding the gradient of the line of best fit to the log intensity values along the averaged A-scan.

In a second experiment, malignant human axillary lymph nodes were taken from two breast cancer patients undergoing axillary clearance. Within 15 min of excision, the fresh lymph nodes were dissected into 2-mm slices and kept hydrated in phosphate-buffered saline solution until they could be imaged. Imaging was performed using a swept-source OCT scanner (ThorLabs, Newton, New Jersey), with central wavelength of $1325 \mathrm{~nm}$, spectral bandwidth of $100 \mathrm{~nm}$, lateral resolution of $15 \mu \mathrm{m}$, and axial resolution of $12 \mu \mathrm{m}$ in air.

The samples were mounted on a glass slide during imaging, and glycerol was used to reduce the refractive index mismatch by displacing any air gaps between the node and the glass plate. After OCT imaging, each sample underwent subsequent histological analysis using hematoxylin and eosin (H\&E) staining in accordance with standard laboratory procedures.

Parametric OCT images were generated for each human tissue sample, and compared against en face OCT images to assess the differentiation of cancerous and healthy tissue. Both sets of images were then validated against the histological H\&E gold standard.

To remove any bias in tissue differentiation due to the selection of window level used for visual display of each type of image, histograms of the intensity values within each of the parametric and en face OCT images were computed. Window levels for each image were set such that the darkest $10 \%$ of pixels were assigned to black, the brightest $1 \%$ of pixels assigned to white, and a linear window level was used for the intervening values, based either on OCT intensity (en face OCT images) or $\mu$ value (parametric OCT images).

\section{Results}

Figure 1 shows the OCT signal decay from the two phantoms, and the calculated line of best fit. The gradient of the line is indicative of $\mu$. Note that signal attenuation is more rapid in the first phantom (Fig. 1, left, $\mu=9.23 \mathrm{~mm}^{-1}$ ) and markedly decreased in the second (Fig. 1, right, $\mu=2.63 \mathrm{~mm}^{-1}$ ) with one quarter the concentration of microspheres.

Figures 2 and 3 show parametric OCT images of two axillary human lymph nodes, with corresponding H\&E histology and matching OCT en face images. Each lymph node contains both areas of malignancy and healthy non-neoplastic tissue. To illustrate the visual differentiation between the two tissue types, several representative healthy areas have been highlighted.

The lymph node shown in Fig. 2 contains metastasis from an invasive ductal breast carcinoma. The histology demonstrates aggregates of malignant cells throughout the node. Some areas of residual, uninvolved (healthy) lymphoid tissue have been circled on both the H\&E histology and parametric OCT images. These areas appear dark in the parametric OCT image, indicating a lower attenuation coefficient in healthy than in malignant tissue. Quantitative studies ${ }^{11}$ have suggested that this is due to changes in size and texture of cell nuclei as
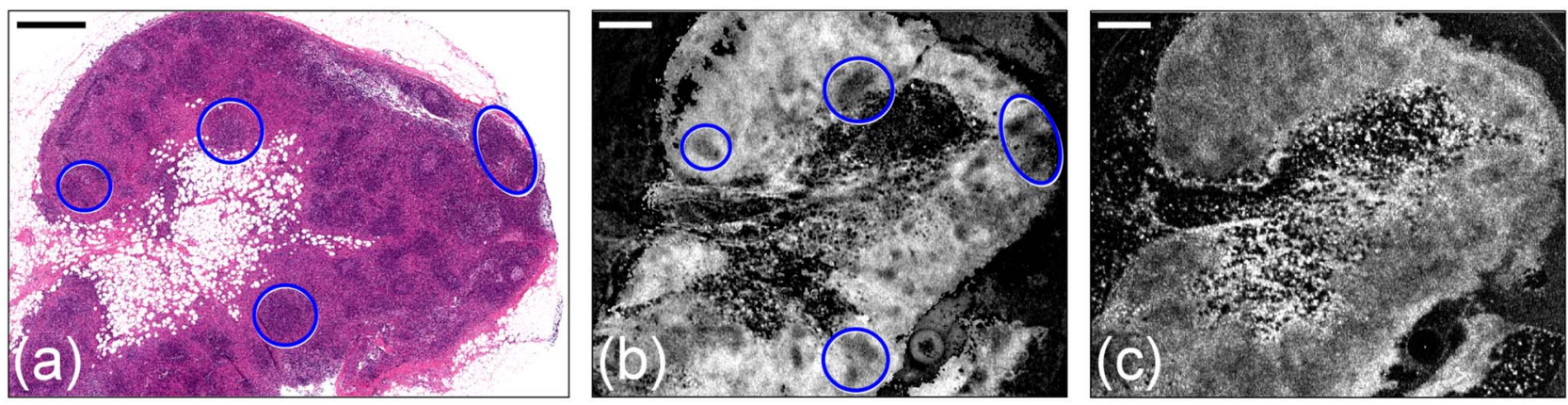

Fig. 3 Involved (malignant) human axillary lymph node with diffuse involvement of the node tissue. (a) H\&E histology; (b) parametric OCT image; and (c) en face OCT image. Circled areas highlight residual, noncancerous cortical tissue. Scale bar=1 mm. 
a result of neoplastic transformation. An optimal OCT en face image is also presented, chosen at the depth that produces the best match to histology. This image shows poorer differentiation between residual cortex and tumor-permeated tissue than is visible in the parametric OCT image.

Figure 3 demonstrates results from a second involved (malignant) lymph node containing metastatic malignant cells from an invasive ductal breast carcinoma. A diffuse pattern of metastatic involvement is visible, with areas of residual uninvolved healthy tissue again highlighted in both the histological and parametric OCT images. Consistent with the first lymph node, areas of residual, healthy cortex exhibit a lower attenuation coefficient than malignant tissue. The parametric OCT image can be seen to give closer correspondence to features visible in histology than is presented by the best matching OCT en face image.

\section{Discussion}

Results presented here demonstrate the ability of a parametric model to improve the differentiation of healthy and cancerous tissue. Through the use of a fully automated algorithm, this has enabled the generation of parametric OCT images that provide a useful visual representation of the underlying mathematical model. Importantly, the use of a parametric model has compressed the 3-D volume of OCT data to a more concise and clinically relevant 2-D representation with improved visual differentiation of areas of malignancy. Such information has great potential to guide needle biopsies of enlarged lymph nodes, where it can be difficult to identify the appropriate region of the node to sample. Through the use of an OCT needle probe, ${ }^{12}$ parametric OCT images could be generated in vivo and preoperatively for assessment of metastasis. Additionally, parametric OCT images of ex vivo lymph nodes could guide the pathologist to sample the optimal area during intraoperative histopathological assessment of sentinel lymph nodes.

These parametric OCT images are based on estimation of a single attenuation parameter $\mu$. Other authors have used different techniques to separately estimate the scattering coefficient $\mu_{s}$ and anisotropy $g$ of scattering. ${ }^{13}$ We note also that more complex models are possible, accounting for the heterogeneity of size and shape of the scatterers in biological tissue. ${ }^{14}$ Such models may provide more sophisticated quantitative measures for tissue differentiation.

The current implementation implicitly assumes that the section of A-scan that is utilized to calculate $\mu$ intersects only a single tissue type. In complex biological structures, this may not be the case, and image segmentation techniques will be required to identify regions of homogeneous tissue, such that the attenuation coefficient of each tissue may be calculated separately for each section of tissue, as proposed in Ref. 15 and demonstrated in Ref. 5.

\section{Conclusion}

In conclusion, a novel form of parameterized OCT image is described, in which the intensity of each pixel is indicative of the tissue's attenuation coefficient across a subset of the A-scan. A fully automated algorithm is developed to generate these images from a 3-D OCT data volume, and the first pub- lished results on malignant human lymph nodes are presented. Uninvolved non-neoplastic lymph node cortical tissue is clearly distinguishable from surrounding malignant tissue containing metastatic cancer, validated against a histological gold standard. These results demonstrate the potential of parametric OCT to differentiate malignant and healthy, uninvolved benign tissues.

\section{Acknowledgments}

We wish to acknowledge the National Breast Cancer Foundation (Australia) for funding R. A. McLaughlin.

\section{References}

1. R. A. McLaughlin, L. Scolaro, P. Robbins, S. Hamza, C. Saunders, and D. D. Sampson, "Imaging of human lymph nodes using optical coherence tomography: potential for staging cancer," Cancer Res. 70(7), 2579-2584 (2010).

2. A. M. Zysk and S. A. Boppart, "Computational methods for analysis of human breast tumor tissue in optical coherence tomography images," J. Biomed. Opt. 11(5), 054015 (2006).

3. M. Mujat, R. D. Ferguson, D. X. Hammer, C. Gittins, and N. Iftimia, "Automated algorithm for breast tissue differentiation in optical coherence tomography," J. Biomed. Opt. 14(3), 034040 (2009).

4. A. Miyazawa, M. Yamanari, S. Makita, M. Miura, K. Kawana, K. Iwaya, H. Goto, and Y. Yasuno, "Tissue discrimination in anterior eye using three optical parameters obtained by polarization sensitive optical coherence tomography," Opt. Express 17(20), 17426-17440 (2009).

5. F. J. van der Meer, D. J. Faber, D. M. B. Sassoon, M. C. Aalders, G. Pasterkamp, and T. G. van Leeuwen, "Localized measurement of optical attenuation coefficients of atherosclerotic plaque constituents by quantitative optical coherence tomography," IEEE Trans. Med. Imaging 24(10), 1369-1376 (2005).

6. C. Xu, J. M. Schmitt, S. G. Carlier, and R. Virmani, "Characterization of atherosclerosis plaques by measuring both backscattering and attenuation coefficients in optical coherence tomography," J. Biomed. Opt. 13(3), 034003 (2008)

7. R. A. McLaughlin, L. Scolaro, P. Robbins, C. Saunders, S. L. Jacques, and D. D. Sampson, "Mapping tissue optical attenuation to identify cancer using optical coherence tomography," in Proc. Med. Image Computing Computer-Assist. Intervention (MICCAI), pp 657664, Springer-Verlag, Heidelberg (2009).

8. S. L. Jacques, D. Levitz, R. Samatham, N. Choudhury, F. Truffer, and D. S. Gareau, "Light scattering in confocal reflectance microscopy," in Biomedical Applications of Light Scattering, A. Wax and V. Backman, McGraw Hill Publishing, New York (2010).

9. T. Collier, D. Arifler, A. Malpica, M. Follen, and R. RichardsKortum, "Determination of epithelial tissue scattering coefficient using confocal microscopy," IEEE J. Sel. Top. Quantum Electron. 9(2), 307-313 (2003).

10. T. Collier, M. Follen, A. Malpica, and R. Richards-Kortum, "Sources of scattering in cervical tissue: determination of the scattering coefficient by confocal microscopy," Appl. Opt. 44(11), 2072-2081 (2005).

11. A. Wax et al., "In situ detection of neoplastic transformation and chemopreventive effects in rat esophagus epithelium using angleresolved low-coherence interferometry," Cancer Res. 63, 3556-3559 (2003).

12. H. Li et al., "Feasibility of interstitial Doppler optical coherence tomography for in vivo detection of microvascular changes during photodynamic therapy," Lasers Surg. Med. 38, 754-761 (2006).

13. R. Samatham, S. L. Jacques, and P. Campagnola, "Optical properties of mutant versus wild-type mouse skin measured by reflectance-mode confocal scanning laser microscopy (rCSLM)," J. Biomed. Opt. 13(4), 041309 (2008).

14. J. M. Schmitt and A. Knüttel, "Model of optical coherence tomography of heterogeneous tissue," J. Opt. Soc. Am. A 14(6), 1231-1242 (1997).

15. J. M. Schmitt, A. Knüttel, and R. F. Bonner, "Measurement of optical properties of biological tissues by low-coherence reflectometry," Appl. Opt. 32(30), 6032-6042 (1993). 